- LANGEVIN, J.P.F. LAF

\title{
Nitin C.A.
}

\section{RÉPONSES}

AUX PROGRAMMES DE 



\section{R ̈ PONSES}

AUX Prograniges DE

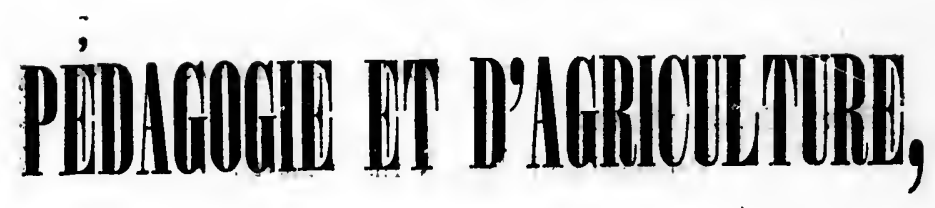

POUR IES DIPLôMEs

D'ECOLE CLCMENTAIRE, D ECOLE MODÈLE ET

D'ACADEMIE,

RÉDIGÉES PAR

Mgr. J LAN hanGEVIN

E'T APPROUVEes PAR LE

CONSEIL DE L'INSTRUCTION PUBLIQUE

TROISILME EDITION

Rimouski :

be L'atelier typographique de ha Voix du Golfe. 


\title{
APPROBATIOA
}

DU

\section{CONSEIL DE L'INSTRUCTION PUBLIQUE}

\author{
Bureau de l'éducatiox, \\ Montréal, 17 Décembre 1862.
}

Retb. M. Lanaevin,

Principal de l'École Normale Laval,

Québec.

Monsiter,

J'ai l'honneur de vous informer que vos "Réponses aux Programmes de Pédagogie et d'Agriculture (nouvelle édition, en franģais et en anglais)" ont été approuvées par le Conseil de l'Instruction publique à sa séance du 10 novembre dernier et que cette approbation a reçu la sanction de Son Excellence le Gouverneur Général, ainsi qu'il appert par minute du Conseil en date du 5 décembre courant.

\author{
J'ai l'honneur d'être, \\ Monsieur, \\ Votre obéissant serviteur, \\ (Signé) P. J. O. CHAUVEAU,
}

Surintendant de l'Education.

Enkegarstre conformément a l'Acte de la Législature Provinciale, en l'année mil huit cent soirante.deux, par Monsieur Jaan Lanagvin, Prêtre, dans le bureau du Registraire de la Prorince du Canada: 


\section{REPPONSES}

\section{a \\ PROGRAMME DE PEDDGOGIE}

POUR LE

\section{DIPLOME D'ÉCOLE ÉLÉMENTAIRE}

\section{CEDULE F. No. 6}

\section{I.}

1. Qu'est-ce que l'éducation?

L'éducation est l'art d'exercer, de développer et de fortifier les facultés du corps, de l'esprit et du cœur des enfants.

2. Qu'est-ce que l'instruction?

L'instruction est l'art d'orner de connaissances l'esprit des enfants.

3. Quels dons naturels sont nécessaires à l'instituteur ?

Les dons naturels, nécessaires à l'instituteur, sont les qualités physiques et intellectuelles qu'il doit passéder.

Les principales qualités physiques, nécessaires à l'instituteur, sont : la clarté de la vue et de l'ouie, une voix convenable, une prononciation distincte, des poumons sains et une santé habituellement bonne.

Les principales qualités intellectuelles sont : une bonne mémoire, un jugement droit, une imagination réglée et un grand tact.

4. Quelles doivent être les qualités morales de l'instituteur?

Les principales qualités morales de l'instituteur sont : la religion, la piété, l'humilité, la gravité, la douceur, la fermeté, la patience, l'activité, la prudence, la discrétion, l'équité, le zelle et la sobriếté. 
La religion lui fuit respecter Dieu et ses uinistres ; la piété le rend exact a ses devoirs religieux; l'humilité le préserve de la suffisance et du pédantisme ; la gravité le fuit respecter des enfants; la douceur lui attire leur affection; la fermetélui fait maintenir la discipline nécessaire ; la patience le rend capable de supporter les défauts des éleves; l'activité lui fait mettre de la vie dans l'enseignement. Par la prudence il veille sur ses démarches; par la discrétion il s'observe sur ses paroles et ses écrits ; par l'équité il évite les passe-droits ; le zèle lui fait remplir ses fonctions avec goût et ardeur; enfin la sobriété le préserve de l'ignoble vice de l'intempérance.

5. Quel doit être le but de l'enseignement ?

Le but de l'euseignement doit être de donner aux enfants des connaissances utiles et pratiques, en même temps que d'exercer et de développer leur intelligence.

6. Faites voir comment l'éducation doit être à la fois physique, intellectuelle et morale.

L'enfant ayant un corps, un esprit et un coeur, l'éducation, pour être complète, doit être à la fois physique, intellectuelle et morale.

L'éducation physique donne de la force et de la souplesse aux membres de l'enfant; elle lui apprend à faire un bon usage de ses sens, à avoir un maintien convenable et à se tenir propre; elle contribue aussi à la conservation de la santé par l'hygiène.

L'éducation intellectuelle doit exercer la mémoire de l'enfant, rectifier son jugement, régler son imagination, et l'habituer a l'observation et à la réflexion.

L'éducation morale réprime chez l'enfant les défauts de carac. - tère, développe les vertus dans son coeur, le plie à la discipline et lui donne les règles du savoir-vivre.

7. Quels sont dans une école les véritables fondements de la discipline?

La véritable discipline dans une école est fondée sur le sentiment du devoir chez les enfants, et sur un grand respect mêló d'affection pour le maître ; et chez celui-ci,"sur le tact, la modération, la gravité et une surreillance enstante. 
8. Comment l'instituteur peut-il parvenir à connâ̂tre seș élèves?

L'instituteur peut parvenir à connaître ses élèves : $1^{\circ}$ en étudiant son propre eœur; $2^{\circ}$ en observant beaucoup les enfants, mais à leur insu; $3^{\circ}$ en les mettant à l'aise afin $q^{\prime}$ 'ils ne songent pas à dissimuler leur caractère.

9. Quelle est la meilleure manière d'apprendre aux enfants a obéir ?

La meilleure manière d'apprendre aux enfants à obéir est de leur faire sentir que ce qu'on leur commande est juste, raisonnable et conforme à leurs véritables intérêts.

\section{II.}

10. Qu'est-ee que le mode d'enseignement individuel ?

Le mode d'enseignement individuel consiste à enseigner séparément à chaeun des enfants d'une ćcole.

11. Qu'est-ee que le mode d'enscignement simultané ?

Le mode d'enseignement simultané consiste à enseigner à la fois a tous les enfants d'une classe.

12. Qu'est-ce que le mode d'enseignement mutuel ?

Le mode d'enseignement mutuel consiste à partager les enfants de l'école par groupes, et à confier ces groupes à la conduite de quelques-uns des élèves les plus capables, qui portent le nom de moniteurs.

13. Qu'est-ce que le mode d'enseignement mixte ou simultané-mutuel ?

J 4 e mode d'enseignement mixte ou simultané-mutuel est celui dans lequel l'instituteur enseigne lui-même successivement a chaque groupe, tandis que les autres groupes sont sous la conduite de moniteurs.

14. Quels sont les avantages que présentent les modes simultané et simultané-mutuel sur les autres ?

Dans les modes simultané et simultané-mutuel, il est plus aisé de maintenir la discipline et d'exciter l'émulation parmi les enfants ; l'instituteur les instruit par lui-même le temps néces-' saire : enfin, les élèves profitent de tout ce qui se fait et de tout ce qui se dit dans la classe. 
15. Comment l'instituteur doit-il rendre son enseignement attrayant ?

L'instituteur doit rendre son enseignenent attrayant, en y mettant lui-même beaucoup d'intérêt, en introduisant de la variété dans le ton et la forme, ainsi que dans les exercices, et en entremêlant ses explications de questions, d'exemples, d'applications et quelquefois d'anecdotes.

16. Faites voir la nécessité de coordonner les maticres à enseigner, même les plus élémentaires.

Il est nécessaire de coordonner les matières à enseigner, afiu qu'il soit consacré à chacune uu temps proportionné à sa nature et a son importance, et que les enfants puissent mieux se préparer pour leurs classes.

17. Pourquoi l'instituteur doit-il procéder du connu à l'inconnu?

L'instituteur doit habituellement procéder du connu ì l'inconnu, parce que c'est la méthode la plus naturelle, qui fait saisir le plus facilement les choses aux enfants, et qui leur apprend le mieux à tirer des conséquences de ce qu'ils savent déjà.

18. Comment faut-il poser les questions aux enfants ?

Il faut poser les questions aux enfants: $1^{\circ}$ d'une manière claire et qui ne cause pas d'équivoque ; $2^{\circ}$ vivement; $3^{\circ}$ sous des formes très-variées ; $4^{\circ}$ d'une façon méthodique $; 5^{\circ}$ plus souvent $d$ un élève en particulier, quelquefois a la classe entière.

19. Quelle est la meilleure méthode pour apprendre aux enfants d connaître les lettres?

La meilleure méthode pour apprendre aux enfants à connuître les lettres est : $1^{\circ}$ de les leur montrar sur un tableau, afin de mieux parler aux yeux ; $2 \circ$ de ue leur en enseiguer que quelques-unes à la fois; $3^{\circ}$ de leur en faire remarquer le son et la forme.

20. Quelle est la meilleure inéthode pour apprendre aux enfants a épeler?

La meilleure wanière d'apprendre aux enfants à épeler est : 10 de leur faire prononcer ehaque lettre ot chayue accent bien distincternent ; 2 = de leur fiure assembled sucecesivement rhapue 
syllabe, puịs le mot entior ; $3^{\circ}$ de les faire épeler beaucoup par ceur ; $4^{\circ}$ de commencer par les mots les plus courts et los plus aisés. On peut encore leur faire prononcer les syllabes sans les épelęr ainsi, d'après la nouvelle méthode.

21. Quelle est la meilleure méthode pour apprendre aux enfants d bien lire?

Pour apprendre aux enfants à bien lire, il faut : $1^{\circ}$ leur.faire faire les liaisons convenables; $2^{\circ}$ leur faire observer les pauses indiquées par les signes de ponctuation; $3^{\circ}$ tendre, non à ce que les enfants en lisent bien long, mais à ce qu'ils lisent correctement ; $4^{\circ}$ no les laisser lire ni trop vite, ni trop lentement ; $5{ }^{\circ}$ leur faire prendre un ton de voix modéré, qui ne soit ni perçant, ni languissant, ni chantant, ni monotone; $6^{\circ}$ leur faire répéter souvent les même phrases.

On doit auşsi tâcher que les enfants lisent avec intelligence, et pour cela leur expliquer la valeur des mots rares ou difficiles, et le sens des phrases, et leur en demander compte ensuite, ce que l'on appelle lecture raisonnée.

22. Quelle est la meilloure méthode pour apprendre la calligraphie aux enfants?

Pour apprendre la calligraphie aux enfunts, il faut : $1^{\circ}$ veiller a la position du papier, du corps, des bras, des mains, des doigts et de la plume; $2^{\circ}$ les accoutumer a avoir une écriture bien régulière sous le rapport de la longueur, de l'inclinaison et do l'espacement des lettres et des mots; $3^{\circ}$ les faire commencer par des barres, puis par les lettres minuscules les plus faciles ; $4^{\circ}$ les faire écrire d'abord en gros, puis en demi.gros, et en dernier lieu, en $f i n ; 5^{\circ}$ les obliger à toujours suivre l'exemple et a conserver leurs cahiers propres; $6^{\circ}$ exiger que tous les devoirs soient écrits avec soin $; 7^{\circ}$ leur donner une bonne expédiéc.

23. Quelle est la meilleure méthode pour enseigner le calcul ?

Pour enseigner le calcul, il est importint : $1^{\circ}$ de commenecr par approndre aux enfants a écrire et a lire les nombres; $2^{\circ}$ do leur faire réciter les tables d'addition, de soustraction, de multiplication, de monnaies, da poids, do mosures, ete. ; $3^{\circ}$ do 
n'avancer que graduellement; $4^{\circ}$ de donner soi-même des exemples sur chaque nouvelle règle, avant de faire résoudre dès problêmes aux élèves ; $5^{\circ}$ enfin, d'accooutumer ceix ci dx être biën méthodiques dans leurs operations de calcul, et a les faire a haute voix, chacun leür tour.

24. Quelle est la meilleure méthode pour enseignér l'orthographe?

La moilleure méthode pour enseigner l'orthographe usuelle àix enfants cst : $1^{\circ}$ de les faire souvent épeler par coeir ; $2^{\circ}$ de leur donner des dictées fréquentes, qu'ils corrigent ímmédiatement sur les indications du maître; $3^{\circ}$ de leur faire reoommencer ces dictées jusqu' $\lambda$ ce qu'elles soient exemptés de fautes.

Quant a l'orthographe grammaticale, on doit : $1^{\circ}$ donner anx enfants a corriger chaque jour quelque exercice correspoìdañt auxx règles de grammaire qu'ils étudient; $2^{\circ}$ leur faire souvent écrire des phrases sur le tableau noir ; $3^{\circ}$ les habituer a analyser, afin qu'ils se rendent bien compte à eux-mềmes de toutes les règles à appliquer.

25. Quelle est la meilleure méthode pour enseigner 'la géographie ?

Pour enseigner la géographie, on doit toujours expliquer d'avance la leģon suivante aux enfants, les habituer a montrer correctement les lieux sur la carte (et non pas seulement les noms), $\grave{a}$ indiquer soigneusement les bornes des pays, le cours des rivières, etc., et a bien connaître les latitudes et les longitudes. Il est aussi très-utile de leur faire répéter souvent les définitions, et de leur faire comprendre l'importance de la géographie.

\section{III.}

26. Quel doit être le but génúral des réoompenses et des punitions?

Le but genéral des réconpenses doit être d'encourager ceux qui les reģoirent, d'exciter l'émulation parmi eux, et d'engager les autres $i$ faire des efforts pour en mériter aussi.

Le but général des punitions doit être de procurer l'amendement du coupable et le bien gúnéral des élèves, en prévenant de aemblables fautes d l'avenir par la crainte des ohátiments. 
27. Quelles sont les fautes qu'on doit surtout punir?

Les fautes qu'ou doit surtout punir, sont : $1^{\circ}$ celles qui sont directement cortraires a la lni de Dieu; $2^{\circ}$ celles qui sont commises avec réflexion et de propos délibéré ; $3^{\circ}$ les fautes d'habitude.

28. Que faut-il surtout récompenser?

Il faut surtout récompenser le trivail, l'upplication, la sagesse, l'assiduité, la docilité ct la conduite régulière.

29. Quels sont les devoirs de l'instituteur envers les parents ?

L'institnteur doit chercher a incpirce aux enfants un grand respect et une grande obéissance envers leurs parents, et ne parler de ceur-oi, devint cux, qu'ave bsamcoup d'egards. Il doit cependant garder son indépendanee pour la conduite de son école, tout en agissant avec prudence et modérition.

30. Quels sont less deroirs des instituteurs envers les autorités civilcs et religieuses?

I'instituteur doit donner l'exemplo de la soumission aux autorités oiviles, en tout ce qui est juste et de leur compétence.

Il doit surtout téwoigner beuucoup do déférneo envers les autorités scolaires en tout ce qui est raisonn:ble, particulièrement a M. le Ministre de l'Instruction Publique, a M. l'Inspecteur d'écoles et a Messieurs les Conmissiires.

L'instituteur doit regarder comine de lio plus laate impertance, sous tous les rupports, de se conserver dins un accorc. pusfuit avco l'autorité religieuse. Il doit done, dans tonters his cirsonstances, faire preuve d'un profund respect pour $\mathrm{i}$. ie 'Nurs ze la paroisse ou il enseigne, demander ses avis, les recevoir avac ciceilité et reconnaissance, et sceondar ses vass pour le bign àcs crefauts.

31. Quels sont lea devoirs dist institutcurs enyers le prizis ?

L'instituteur doit éviter do prendro awcurso navi', nst:ve dins les divisions qui peuvent exister dins la parcisas cin il est plnes. Il doit en même temps s'efforcer d'icquérir l'estimie génériale par une conduite irréprochable, uno grande modestie et une grande politesse, et de so rendre utile dius la limite de ses connaissances et des moyens il sa disposition. 


\section{RHTONAT}

AU

\section{PBOGBAIIIE DE PEDGCOGE}

POUR LA

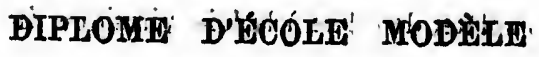

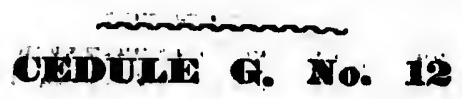

\section{I.}

1. Qu'est-ce que la PEdagogie ?

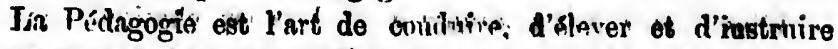

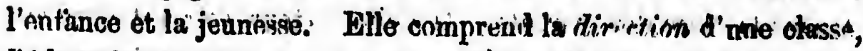
l'éduccation et l'iustricction; et renferme nutis partio theoriques ot ube partie printiquie.

2.: Quels sont les fondements et les prinsipes de rette seierice?

Les principes de la Pédagogie sont fondés sur la cornaissarane de la nature des enfants et sur l'experience des meillentrs maitres

3. Quelles sost les vertus partioulitios que doit posseder l'institutear ?

Parmi les vertus particulières que dnit posséder tai instituteur, on doit compiter : une granide innorence de moours, lo probite, le desintéressement et lo dóvouenienit:

4. Qu'est-ce que l'éducution?

L'éduoation est l'art d'exercer, de développer et de fortifiter les facultés du corps, de l'esprit et du coeur des enfants. 


\section{Qu'est-re que l'instruction ?}

L'instruetion est l'art d'orner de connaissances l'csprit des enfants, par le moyen de l'enscignement.

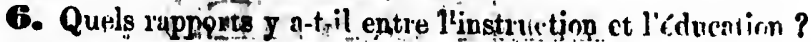

Il y a dis rapports tris-étroits entrel'iustruction et l'ćdncintion,

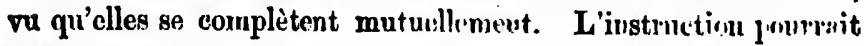
même devenir furt perniesicuses. si elle n'itrit acronlpignen do

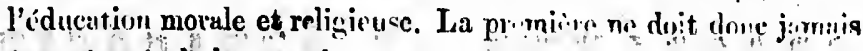
êtru séparáq de la sutendle.

7. Pourquoi l'éducation doit-elle itte a la this ghy:iqua. intellectuclle ct moriale?

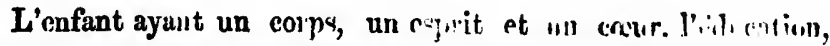

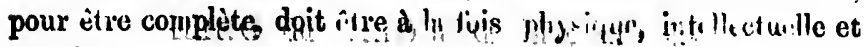
morale.

8. Qu'est-ce que l'rillucation phr virue ?

L'éducation physique dur.ne de la forpe et de la snuphlone aux

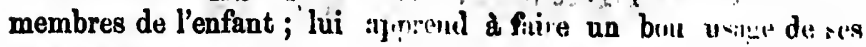
sens, a avoir un maintien combinible et a se tenir propire; elle contribue aussi a la conservation de la sinté par l'hy winne.

8. Dins quelle mesure l'instituter doit-il s'orcupur de l'ciluoation physique?

L'instituteur doit s'ocsuper de liedungtion physiqu. camme tenant la place des parents. Il drit surtout ruic a dunn!r aux onfapts un bon maintion, a leur en inger a binn no pluyer leurs sens, et a dirigor les jenx auxquels il se livrent entre les elnsses.

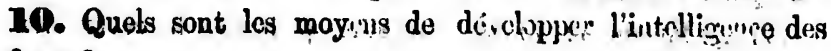
enfants ?

Pour développer l'intelligñuge des enfants il faut les accoui umer a bien observer et a rendre ri:inon de ce qu'ils apprennent.

11. Jusqu'd quel point l'instituteur doit-il dúve'unp r la sensibilití ohez les enfants ?

L'inotituteur doit se gurder de trop dévşinpper la sensibilité chez les enfants ; il doit au enptraire s'efforcer de la moulúrcr par la réflexion et l'éloignement de la mollessse.

19. Comment doit-on fortifier la volonté chez les enfants ?

- On doit fortifior la volonté chez les enfants en les accoutumant 
a se plier à la disoipline, â résister à leura 'ponchants naturels, et a niontrer de la force 'morale, de l'cnergie de caractère dans les circonstances critiques ou pénibles.

13. Quelles sont les bases de l'éducation notale ?

L'éducation morale a pour bases la loi de Dicu, le sentiment du devoir et do l'honneur.

\section{II.}

14. Quel doit être le but de l'enscignement ?

Le but de l'enseignement doit être 'de douner anx enfants des cummaissances utiles et pratiques, en même temps 'que d'exorcer et do développer leur intelligence.

155. 'Fiites voir l'utilite qu'il y a pour l'instituteur d'avoir un plän d'études pour son école.

Il est nćcessaire pour l'instituteur d'avoir un plan d'études, afin qu'il consacre à chaque matière un temps convenable, que les exercices "soient "mieux variés, qu'il perde moins de temps en passant de l'un a l'autre,"enfin que les enfants së prẻparent mièux pour leurs classes.

16. Quelles' qualités doit posséder ce plan?

Le plan d'études adopté par un instituteur pour son école ne doit pas être trop compliqué, doit accorder à chaque matière le temps que requièrent sa nature et son importance, et être proportionné au nombre d'années que les enfants passent à l'école.

17. Quelle préparation l'instituteur doit-il toujours apporter à ses classes?

L'instituteur doit apporter à ses classes deux sortes de prépation, la préparation eloignée et la préparation prochaine.

Par préparation éloiguée, il faut entendre le soin de l'instituteur a consacrer régulierement ehaque jour un certain temps a l'étude; surtout à celle des matières qu'il est appelé à enseigner.

Par préparation prochaine, on entend le soin que doit apporter l'instituteur à prévoir la leçon suivante, la manière de l'expliqner, les exemples destinés al l'éclaireir, et les applications à donner aux enfanits.

18. Quels sont les 'defauts que l'instituteur doit surtout bannir de son école ? 
Les principaux défauts qu'un instituteur doit bannir de son Sivle; sont : l'immou alité, le meturouge, la dissimulation, le vol, la paresse, l'orguvil, l'insuborüination, la malpropreté, la grossièreté et la dissipation.

19. Quelles sont, les busiss de la váitable discipline?

La véritable diseipline dius une école est fondée sur le sẹntiment du devoir chez les enfänts, et sur un grand respeet mêlé d'affection pour le maître ; et clinz celui-ci, sun le tact, la modération, la gravité et une survellance, constante.

20. Quels sont les devuirs de l'instituteur pendunt la clusse ?

Pendant la classe, l'iustituteur, doit avoir un maintien digne, exercer une surveillance continuelle, tenir les enfants toujours occupés, ne pas perdrc un instant et conserver une parfaite égalité d'humeur.

21. Comment l'insticuicur, peut-il wi cuir $\left\{\begin{array}{l}\text { l'obéissauce ? } \\ \text { l'ordre et le silence ? } \\ \text { la propreté ? } \\ \text { la modestie ? } \\ \text { la politesse ? }\end{array}\right.$

I. La meilleure manière ' d'upprendre èux enfants à obcuir est de leur faire sentir que ce qu'on leur commands, est juste, raisonuuble et onnforme a leurs véritables intérêts.

- II. Pour obtenir l'ordre et le silence, l'instituteur doit avoir lui-même beaucoup de méthode et de modération, parler ordinairement d'une voix médioore, tenir les enfants toujours ocenpés et attentifs, et récompenser la sagesse.

III. Pour obtenir la propreté des enfants, l'instituteur doit tenir sa classe très-propre, donner lui-même l'exemple de la propreté, en faire comprendre l'avantage pour la santé, faire chaque jour: un examen de propreté pour la tête, les mains.et les habits, enfin exiger que les enfunts soient très-scigneux de leurs livres et de leurs cahiers.

IV. La modestie ou humilité peut. s'obtenir des enfants, en leur rappelant que les talents viennent de Dieu, qui.en depmandera compte un jour, en leur faisant voir les eharmes de la modestie, et en acoompagnant les élogess et les récompenses de quelques paroles propres a prévenir l'orgueil. 
Quant aux bonnes mœurs des enfants, qu'on appelle aussi modestie, on les conserve en veillant sur leurs compagnies, leurs. discours et leurs lectures, et en les engageant it se rappeler sans cesse la présence de Dieu.

V. L'instituteur peut obtenir la politesse des enfants, en se montrant lui-même'très.poli envers eux, en leur expliquant les règles du savoir-vivre, en leur reeommandant beaucoup d'égards les uns pour les autres, enfin en exigcant d'eux une grande civilité dans les actions et les paroles.

22. Quels sont les moyens de rendre les elères attentifs pendant la classe ?

Les meilleurs moyens de rendre les clèves attentifs pendant la classe sont : $1^{\circ}$ de varier souvent les exereices; $2^{\circ}$ de ehauger fréquemment la forme et le ton des explications; $3^{\circ}$ de faire des questions il l'improviste, tantôt individuellement, tantôt simultanément ; $4^{\circ}$ de s'adresser aux yeux des enfants, autant $q^{\prime} u^{\prime} \mathbf{u}$ leurs oreilles; $5^{\circ}$ de leur promettre comme prix de leur attention, quelque aneedote intéressante ou quelque leçon de shoses à la fin de la classe ; $6^{\circ}$ de rendre l'enseignement attrayant en $\mathrm{y}$ mettant soi-même beaucoup d'intérêt.

\section{III.}

23. Comment doit se comporter l'instituteur dans une classe composée d'élèves forts et d'élèves faibles ?

L'instituteur ne doit pas passer trop rapidement d'une chose it une autre, parce que les élèves faibles ne pourraient le suivre; il ne doit pas non plus avaneer trop lentement, vu que ce serait une injustice envers les élèves forts : il doit se proportionner à la capacité du plus grand nombre. S'il y avait moyen même de partager la classe en plusieurs divisions, il devrait le faire.

24. En quoi consiste la forme d'exposition ?

La forme d'exposition consiste a expliquer un sujet aux enfants on allant du simple au composé, des principes aux conséquences : c'est ce que l'on appelle encore symthèse.

25. En quoi consiste la formo d'invention ?

La forme d'invention consiste it fuire trouver les choses aux enfants eux-mênes, al les alceoutumer à remonter des conséf uteuces 
aux principes, des applications aux règles : c'est ce que l'en appelle aussi analyse.

26. Quels sont les avantages de chacune de ces formes ?

La forme d'exposition convient mieux aux premières explication sur une maticre, en donne des notions plus suivies, et exerce davantage la mémoire.

Par la méthode analytique on s'assure mieux si les enfants ont bien compris ce qu'on leur a explique. Cette forme les oblige plus à raisonner, et exerce ainsi davantage le jugement.

27. Comment doit-on coordonner les matières qu'on enseigne?

Les matières qu'on enseigne, doivent être coordonnées selon la nature de l'école que l'on tient, l'âge et la capacité des enfants, l'importance de chaque branche d'étude, et le temps qu'elle exige, soit par jour, soit par semaine.

28. Quels avantages y a-t-il à procéder du connu'a l'inconnu, du simple au composé?

L'instituteur doit habituellement procéder du connu à l'inconnu, parce que c'est la méthode la plus naturelle, qui fait saisir le plus facilement les choses aux enfants, et qui leur apprend le mieux ì tirer des conséquences de ce qu'ils savent déjà.

29. Quelles sont les qualités requises pour bien exposer les unaticres ?

L'exposition des matières doit être : $1^{\circ}$ claire, e'est-à-dire pouvant être facilement comprise; $2^{\circ}$ méthodique et graduée, c'est-i-dire donnée dans un ordre suivi et naturel ; $3^{\circ}$ mise a la portée des enfants, par rapport à leur âge et d leur avancement ; $4^{\circ}$ conforme al la classe d'école que l'on tient; $5^{\circ}$ interrompue par des questions faites à propos ; $6^{\circ}$ enfin, rendue compréhensible et intéressante par des excmples, des exercices et des applications.

30. Quelles sont les qualités requises pour bien questionner ?

Il faut poser les questions aux enfants : $1^{\circ}$ d'une manière claire et qui ne cause pas d'equiroque ; $2^{\circ}$ vivement $; 3^{\circ}$ sous des formes très-varices ; $4^{\circ}$ d'une façon méthodique ; $5^{\circ}$ plus souvent à un élère en particulier, quelquefois il la classe entière. 
31. Comment doit-on exereer chez les enfunts la mémoire des choses et la mémoire des mots ?

Pour ezercer chez les enfants la mémoire des choses, il faut les habituer a rendre compte des lectures qu'ils font a haute voix ou qu'ils entendent pendant la elasse, aussi bien que des objets qu'ils rencontrent, et qu'ils doivent s'accoutumer ì remarquer.

Pour exercer chez les enfants la mémoire des mots, il est utile de leur faire apprendre régulièrement par coour des leçons d'une longueur raisonnable, et assez souvent des morceaux qui aient de l'intérêt pour eux, comme une pièce de poésie proportionnée à leur âge. Mais cette mémoire des mots doit toujours être accompagnée de la mémoire des chóses.

\section{$\mathbf{I V}$.}

32. En quoi consiste le mode individuel ?

Ise mode individuel consiste à enseigner sćparément $i$ chacun des enfants d'une école.

33.. En quoi consiste le mode simultané ?

Le mode d'enseignement simultané consiste à euseigner à la fois a tous les enfants d'une classe.

34. En quoi consiste le mode mutuel ?

Le mode d'enseignement mutuel consiste ì partager les enfunts de l'école par groupes, et à confier ces groupes à la conduite de quelques-uns des élèves les plus capables, qui portent le nom de moniteurs.

35. Quels sont les avantages et les défauts de claque mode ?

Le mode individuel a pour avantage que l'instituteur peut plus facilement se proportionner a la capacité de chaque élève. Les défauts en sont : qu'il est plus fatigant pour le maître; qu'il ne permet $a$ celui-ci de donner que très-peu de temps à chaque enfant; qu'il présente beaneoup de difficultés pour le maintain de la discipline; enfin qu'il ne peut aucunement exciter l'émulation parmi les élères.

Le mode simultané a pour principaux avantages : $1^{\circ}$ d'obliger le maître à moins de répétitions; $2^{\circ}$ de faire profiter les enfants de tout ce qui se dit et de tout ce qui se fait dans la classe ; $3{ }^{\circ}$. d'ex citer puissamment l'émulation parmi enx. Il a l'inconvénient 
que les:o plications ne sont pas proportionnées ù la capacite de tous les élèves.

Le mode mutuel enfin a pour avantage de tenir tous les enfants de l'école continuellement occupés à la fois. Mais il présente le grave inconvénient : $1^{\circ}$ que les enfants des dirers groupes sont dirigés simplement par des moniteurs, qui souvent sont soit trop peu instruits, soit trop légers, soit trop enflés de leur petite autorité, soit enfin peu équitables; $2{ }^{\circ}$ que les moniteurs n'ont pas assez de temps pour etudier eux-mêmes.

36. Quels sont les caractìres essentiels d'une bonne méthode?

Une bonne methode doit avoir pour effets de tenir les enfants sages et attentifs, d'exciter parmi eux une louable émulation, de ménager le temps, ainsi que la santé de l'instituteur, tout en faisant faire aux cleves des progrès constants.

37. Quels est le mode qui convient lo mieux a la plupart des Éoles?

Le mode qui convient le micux à la plupart des écoles, est celui qu'on appelle mixte, et dans lequel l'instituteur enseigne lui-même successivement à chaque groupe, tandis que les autres groupes sont sous la conduite de moniteurs.

Dans ce mode, qu'on appelle aussi simultané-mutuel, il est plus aisé de maintenir la discipline et d'exciter l'émulation parmi les enfants ; l'instituteur les instruit par lui-même le temps nécessaire; enfin les ćlèves profitent de tout ce qui se fait et de tout ce qui se dit dans la elasse. Seulement le maître doit préparer soigneusement les moniteurs ì remplir leur fonctions.

38. Quelle méthode peut on suivre pour apprendre aux enfants à connaître les lettres ?

La meilleure méthode pour apprendre aux enfants à connaître les lettres est : $1^{\circ}$ de ne leur en enseigner que quelques-unes at la fois ; $2^{\circ}$ de les leur montrer sur un tableau, afin de mieux parler aux yeux; $3^{\circ}$ de leur en faire bien remarquer le son et la forme.

39. Quelle méthode faut-il suirre pour apprendre aux enfints à bien Epeler? 
La meilleure manière d'apprendie aux enfants à épeler (suivant l'ancienne méthode) est : $1^{\circ}$ de leur faire prononcer chaque lettre et chaque accent bien distinctement; $2^{\circ}$ de leur faire assembler successivement chaque syllabe, puis le not entier; $3^{\circ}$ de commencer par les mots les plus courts et les plus aisés ; $4^{\circ}$ de les faire épeler beaucoup par cour. On peut encore leur faire prononcer les syllabes sans les épeler ainsi, d'après la nouvelle méthode.

40. Quelle méthode faut-il suivre dans l'enseignement de la ealligraphie?

Pour apprendre la calligraphie aux enfants, il faut: $1^{\circ}$ veiller a la position du papier, du corps, des bras, des mains, des doigts et de la plume; $2^{\circ}$ les accoutumer à avoir une écriture bien régulière sous le rapport de la longueur, de l'inclinaison et de l'espacement des lettres et des mots; $3^{\circ}$ les faire commencer par des barres, puis par les lettres minuscules les plus faciles ; $4^{\circ}$ les faire écrire d'abord en gros, puis en demi-gros, et en dernier lieu en $f i n ; 5^{\circ}$ les obliger à toujours suivre l'exemple et à conserver leurs cahiers bien propres; $6^{\circ}$ exiger que tous les devoirs soient écrits avec soin.

41. Commont doit-on apprendre aux enfants les éléments de l'orthographe ?

La meilleure méthode pour enseigner l'orthographe usuelle aux enfints est : $1^{\circ}$ de les faire souvent épeler par cour ; $2^{\circ}$ de leur donner des dictées fréquentes, qüils corrigent immédiatement sur les indications du mître; $3^{\circ}$ de leur faire recommeneer ees dictées jusqu'ì ce qu'elles soient exemptes de fautes.

Quant à l'orthographe grammaticale, on doit : $1^{\circ}$ donner aux enfants à corriger chaque jour quelque exercice correspondant aux règles de grammaire qu'ils étudient; $2^{\circ}$ leur faire souvent écrire des phrases sur le tableau noir ; $3^{\circ}$ les habituer ì analyser, afin qu'ils se rendent bien compte à eux-mêmes de toutes les règles at appliquer.

42. A quelle méthode l'enseignement de la grammaire et celu. de l'arithmótique peurent-ils être soumis ? 
La granmaire peut s'enseigner par syithèse ou par analyse. On définit d'abord clairement la partie du discours dont il s'agit, en expliquant soigneusement tous les mots que renferme la définition. Puis on donne successivement les différentes règles, ev les éclaircissant par des exemples bien choisis et des exercices convenablement gradués.

Pour s'assurer que les élèves ont compris et retenu les règles, on les habitue ensuite à l'analyse grammaticale, qu'on leur fait faire d'une manière graduée et méthodique. Lorsqu'ils sont plus âgés et plus avancés, il est fort utile de leur enseigner l'analyse logique.

Pour enseigner le calcul, il est important : $1^{\circ}$ de commencer par apprendre aux enfants a écrire et $a$ lire les nombres ; $2^{\circ}$ de n'avancer que graduellement; $3^{\circ}$ de donner soi-même des exemples sur chaque nouvelle règle, avant de faire résoudre des problèmes aux élèves; $4^{\circ}$ enfin, d'accoutumer ceux-ci à être bien méthodiques dans leurs opérations de calcul et al les faire a haute voix, chacun leur tour.

43. Quelle est l'importance du calcul mental ?

Le calcul mental est bien important pour exercer la mémoire des enfants, les accoutumer ì retenir les dates, et leur faire faire promptement beaucoup d'opérations usuclles et pratiques en arithmétique.

44. Dans l'enseignement de la géographie et de l'histoire fautil s'attacher à l'exereice de la mémoire ou de l'intelligence?

Dans l'enseignement de la géographie et de l'histoire il faut exercer aussi bien l'intelligence que la mémoire. Ainsi, en géographie, on demandera aux enfants la route a suivre pour aller de tel lieu à tel autre, les divers objets d'échange entre certains pays, la position des montagnes d'après lo cours des rivières, etc. Les cartes muettes sont préférables pour les élèves déja avancés.

Pour bien enseigner l'histoire, il faut: $1^{\circ}$ non seulement la faire apprendre par coeur aux onfants, mais aussi les habituer à en rendre compte a leur manière; $2^{\circ}$ leur faire indiquer sur la carte tous les lieux mentionnés dans la leçon $; 3^{\circ}$ les accoutumer a distinguer les faits principaux arec leur's dates, des faits secon- 
daires ; $4^{\circ}$ leur faire faire des réeapitulations assez fréquentes, afin qu'ils lient bien les fairs les uns aux autres.

45. Qu'est-ce que les les leçons de choses et quel peut être le sujet de ces leçons?

On entend par leçons de choses des détails donnés par l'instituteur sur différents objets et entremêlés de questions adressées aux élèves. Ces leçons peuvent avoir pour principal sujet les objets les plus ordinaires darq une école, dans une maison quelconque, dans les travaux de l'agriculture, aussi bien que des êtres appartenant aux différents règnes de la nature, tels qu'animaux, plantes, minéraux, ete.

46. Comment l'instituteur peut-il inculquer aux enfants des connaissances sur les choses usuelles?

L'instituteur peut inculquer aux enfants des eonnaissances sur les choses usuelles en donnant des explications sur ees ehoses : 10 lorsque le nom s'en rencontre dans les livres de lecture, dans la géographie, l'histoire, etc. ; $2^{\circ}$ dans les promenades qu'il peut faire quelquefọis avee eux $; 3^{\circ}$ dans les leģons de choses.

47. Quel doit être le but des récompenses et des punitions?

Le but général des récompenses doit être d'encourager ceux qui les reçoivent, d'exciter l'émulation parmi eux, et d'engager les autres a faire des efforts pour en mériter aussi.

Lo but général des punitions doit être de procurer l'smendement du coupable et le bien général des élèves, en prércuant do semblables fautes a l'avenir par la crainte des ehâtiments.

48. Dans quelle mesure doit-on se servir des réeompenses et des punitions?

On doit se servir des récompenses et des punitions avec paroimonie; des premic̀res, comme témoignages de satisfaction et encouragement it aceomplir fidelement lo devoir ; des secondes, comme mesure extrême et dernier moyen de prévenir ou de réprimer le mal.

49. Qu'cntendez-vous par punition positive et par punition naturello ?

Par punition positice j'entends celle qui est imposce par la 
volonté de l'instituteur; par punition naturelle, celle qui résulte nécessaircmont d'une faute, par exemple : la honte, lignorance, la perte de l'estime, le chagrin des parents, etc.

50. En punissant faut-il considérer lintentiou ou l'action extéricure du coupable, et pcurquoi ?

En punissant il ne faut fas scule ncont consiáérer l'action extéricure du coupable, mais cncore et surtcut l'intention, autant qu'on peut la connaîtıe, fuisque celle-ci seule détermine la moralité de l'action et le dégré de culpabilité.

51. Faites roir sil ne faut laisser aucune faute impunie.

Il faut laisser un bcn ncribre de fautes inpunies, c'est-à-direcelles qui provienncnt uniquement de la légèreté, de l“irréflexion, et qui ne sont pas propres à introduire le désordre dans la classe.

52. Quand et comment doit-on imposer des punitions?

Les punitions doivent être : $1^{\circ}$ assez rares pour faire impression ; $2^{\circ}$ données avec sang.froid et modération ; $3^{\circ}$ de nature à ne pas blesser la modestie et à ne pas nuire à la santé des cnfants.

53. Qu'est-ce qu'il faut récompenser ?

Il faut surtout récompcnser" le travail, l'application, la sagesse, l'assiduité, la docilité et la conduite régulière.

54. Quelles récompenses faut-il donner?

Il faut donner comme récompenses aux enfants : $1^{\circ}$ des témoignages d'approbation; $2^{\circ}$ des marques de confiance; $3^{\circ}$ de bonnes places, de bonnes not is, de bons points ; $4^{\circ}$ l'inseription sur une liste d'honneur ; $5^{\circ}$. quelque signe do distinetion $; 6^{\circ}$ des images et des livres proportionués au genre d'éeole et $i$ l'arancement des enfants. On doit leur appreadre ì estimer ees objets, non à cause de leur valeur intrinsèque, mais par rapport au motif qui les leur fait donner.

55. Quund ct comment faut-il donner des récompenses?

Il fuut donner des récomipenses assez souvent pour encourager les enfants, mais pas assez pour qu“elles soient indifférentes a leurs yeux.

En récompensant on doit éviter soigneusement de eommettre des passe-droit, de provoquer parmi les enfints des sentiments de bainc ou d'cuvie, cnfin de surexciter lcur amour-propre. 


\section{VI.}

56. Qucls sont les objets dont une naison d'école doit être pourvue?

Une maison d'école doit être surtout pourvue: $1^{\circ}$ de moyens d'aérer la classe et d'y maintenir une température modéréc ; $2^{\circ}$ d'ouvertures en nombre suffisant pour la bien éclairer $; 3^{\circ} \mathrm{d}$ 'un crucifix ; $4^{\circ}$ d'une estrade, d'un siége et d'une table pour l'instituteur $; 5^{\circ}$ de banes avec dossiers et de tables pour les élèves; $6^{\circ}$ de tableaux noirs et de cartes géographiques ; $7^{\circ}$ d'une pendule et d'une clochette; $8^{\circ}$ de globes, pour les ́́eoles modeles; $9^{\circ}$ de erochets pour pendre les habits et les coiffures.

57. Quels sont les devoirs de l'instituteur dans ses rapports avec les commissaires?

L'instituteur doit témoigner beaucoup de déférenee evers les commissaircs en tout ec qui est raisonnable, et leur fournir sur son école tous les rensciguements requis.

58. Quels sont les devoirs de linstituteur dans ses rapports avee lc curé ?

L'instituteur doit, dans toutes les cireonstances, faire preuve d'un profond respeet pouś MI. le curé de la paroisse où il enseigne, demander ses avis, les reeevoir avee docilité et reconnaissance, et seconder ses vues pour le bien des enfints.

59. Quels sont les devoirs de l'instituteur dans ses rapports avec les parents des élìves.

L'instituteur doit ehereher it inspirer aux enfints lin grand respeet et une grande obéissance envers leurs parents, et ne parler de eeux-ci devant enx qu'avee beaconp d'égards. Il doit eependant garder son indépendance pour la condnite de son école, tout en agissant avce prudenee et modération.

60. Quels sout les devoirs de linstituteur dans ses rapports avec le publie?

L'instituteur doit éviter de prendre aueune part active dans les divisions qui peuvent exister dims la paroisse où il est placé. H doit en mêne temps s'efloreer d'acquérir l'estime généralo par une execllente eonduite et une grimle politesse, et de se rendre ntile daus la limite de ece comminissances ct des moyens ì sil disposition. 


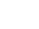




\section{REPONSES}

AU

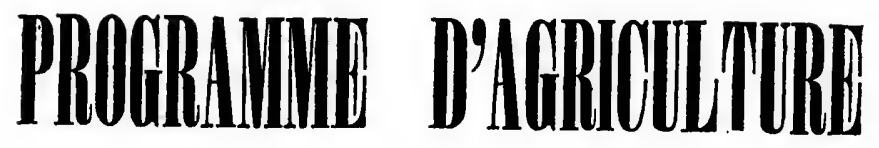

POUR LE

DIPLOME D'ÉCOLE MODÈLE

\section{CEDULE G. No. 13}

I.

1. Q'est-ee que l'Agriculture ?

I'Agriculture est l'art de cultiver la terre d'une manière avantageuse et économique.

2. Quels avantages présente l'Agriculture ?

I'Agriculture présente pour principaux avantages, d'être une nceupation : $1^{\circ}$ très-importante $\mathrm{a}$ un pays; $2^{\circ}$ salubre; $3^{\circ}$ solide et sûre $; 4^{\circ}$ indépendante et honorable; $5^{\circ}$ très-favorable it la conservation des mœurs.

3. Que faut-il connaître pour être bon agriculteur ?

Pour être bon agricultcur il faut connaître $1^{\circ}$ la lecture et l'écriture; $2^{\circ}$ les éléments de l'aritlmmétique; $3^{\circ}$ les premiers éléments de la plyssique, de la mécanique, do la chimie et de l'histoire naturelle. 
Il faut encore connaître les diverses espèces" de terres et de semences, et l'usage des instruments perfeetionnés.

Il faut de plus une conduite régulière, de la santé, de l'aetivité, de la prudence, de l'éeonomie, de la persévérance, de l'esprit d'ordre et d'observation.

4. Pourquoi faut-il distinguer les différentes especes de terres qui composent un sol ?

Il est important de connaître les différentes espèces de terres qui composent un sol, afin de savoir l'usage qu'on en doit faire, la manière de l'assainir et de le préparer, et les sortes de plantes que l'on doit y cultiver de préférence.

5. Indiquez les avantages de chaque espèce de terre, et les plantes qui viennent le mieux dans chaque terre.

On appelle terre forte celle où domine la glaise ou argile; elle est tenace et froide; on la reconnaît ì ce qu'elle se crevasse à la sécheresse, et que l'eau séjourne à sa surfaee. Les avantages en sont : qu'clle garde micux la fraieheur, offre aux racines une base plus solide, et eonserve plus longtemps la richesse que lui ont communiquée les engrais. En revanche, elle retient trop l'humidité dans les temps de pluie, se crevasse et se durcit trop dans les temps de sécheresse. Le blé, l'avoine, les fèves et les betteraves $\mathrm{y}$ viennent bien, ainsi que le trèfle.

On appelle terre légère celle qui est formée surtout de sable ou de carbonate de chaux (matière dont on pent extraire de la chaux). Les avantages d'un terrain sablonneux sont : qu'il se ressuie plus vite, que les plantes y lèvent et y mûrisrent plus tôt, et que les cultures y sont faciles et moins coûteuses. Les inconvénients en sont : de s'assécher trop rapidement, de retenir peu les matières fertilisantes, et de trop exposer les plantes aux variations brusques de température. L'orge, le seigle, le sarrasin, les navets, la pommes de terre (patatcs) y réussissent particulièrement.

Les avantages du calcaire dans le sol sont : de rendre les terres fortes plus meubles, plus friables, et conséquemment plus faciles ì cultiver, et de donner aux terres légères plus de consistanee et par lì même d'en faciliter aussi la culturc. Lu carbonate de 
chaux d'ailleurs augmente la quantité de certains produits. L'orge et le sainfoin réussissent bien dans les terrains calcaires.

Enfin la meilleure terre, ou terre franche, est celle qui renferme de l'argile, de la silice (sable et cailloux) et du carbonate de chaux en proportions convenables, avee environ un douzième d'humus ou terreau. On appelle ainsi une substance brune ou noirâtre produite par la décomposition des matières animales ou végétales.

\section{II.}

6. Quels sont les principaux proeédés pour améliorer le sol ?

Les principaux proeédés pour améliorer le sol sont :

$1 \circ$ le defrichement, qui consiste à mettre en état de culture, soit un terrain abandonne, soit un bois ;

$2^{\circ}$ l'épierrement, qui consiste à débarrasser le terrain des pierres dont-il est encombré ;

$3^{\circ}$ l'ecobuage, qui consiste it enlever par tranches la croûte supérieure du sol et à la brûler ;

$4^{\circ}$ l'assainissement, qui cousiste à délivrer le sol des eaux surabondantes ou stagnantes ;

$5^{\circ}$ enfin, les amendements, qui consistent dans le mélange avec le sol de certaines substances qui (comme la chaux, la marne siliceuse et le sable) le rendent meuble, s'il est trop compacte ; ou qui (comme l'argile, la marne argileuse et la chaux) le rendent plus ferme, s'il est trop meuble.

7. Quelles sont les différentes matières qui peuvent servir d'engrais ?

Voici les différentes matières qui peuvent surtout servir d'engrais (substances destinées à enrichir lo sol) : $1^{\circ}$ certaines plantes qu'on enfouit avant qu'elles ne soient mûres, ce qu'on nomme engrais verts ou engrais végétaux; $2^{\circ}$ les tourteaux qui viennent du lin, après qu'on en a extrait l'huile ; $3^{\circ}$ les excréments et les urines, qu'on appelle engrais animaux; $4^{\circ}$ les fumiers d'étable, ou engrais mixtes; $5^{\circ}$ les cendres; $6^{\circ}$ les composts, formés de chaux et de marne mêlées par couches avec des débris de toute nature. 
5. Pourruoi faut-ii distinguer ies fumiẹrs chauủs ü arce les fumiers froids ?

Il fout distinguer les fumiers chauds d'arec les fumiers froids, paree gre les uns et les autres ne conviennent pas également à toutes les especes de terrains. ILes premiers (excrements de l'homme, des roiriiles. des cheraux et des moutons), conriennent aux terres fortes et froirios; los foconds (excréments des bêtes ì cornes), aux terres stblouncisens ef legires.

D. Quels sont les soins à conner au fumicr pour qu il soit bon ?

Pour que le fumier soit bon, il faut: $1{ }^{\circ}$ fuire bien attention it la litière des animaux ; $2^{\circ}$ placer le fumier sur un paré, ou du moins sur une couche de terre glaise ; $3^{\circ}$ avoir soin que le jus du fumier ne se perde pas, mais l'employer $\grave{a}$ arroser souvent le tas ; $4^{\circ}$ ne pas entasscr le fumicr $\dot{a}$ une trop grande hautcur ( 6 i 7 pieds suffisent) ; $5^{\circ}$ prendre garde qu'il ne soit pas lare par l'eau du toit ou de quelque ruisseau ; $6^{\circ}$ quand le tas a la hauteur voulue, le couvrir d'une couche de terre, pour empêcher l'évaporation des principes fertilisants.

10. Quelle est l'utilité du plâtre?

le plâtre est utile : $1^{\circ}$ pour être mêlé yar couches au fumier, afin d'en conserver les bonnes qualités; $2^{\circ}$ pour êtro répandu en poudre sur le sol, surtout sur les terrains secs et chauds, ou sur les pois, le sainfoin, le trèffe, ete., quand ils commencent à pousser, pour activer la végétation, coume stimulant.

11. Qu'est-ce que l'assolement : quels en sont les avantages ?

On appelle assolement l'ordre dans lequel se succèdent les direrses productions d'un même terrain : il peut être de trois, de quatre,...de huit, de neuf ans, etc. Cette succession de produits est bien nécessaire, parec que parmi les plantes, les unes servent $\grave{a}$ amcublir le sol, d'autres ì le nettoyer ; quelques-unes sont améliorantes, d'autres épuisantes, etc. Il faut que l'assolement ait pour effet de rendre au sol ce qu'on lui a enlevé.

12. Quel est le meilleur plan d'assolement ?

Ise meilleur plan d'assolement est celui qui réunit les conditions suivantes : $1^{\circ}$ approprier les récoltes au climat, ì la nature $d u$ sol et aux ressources dont on dispose; $3^{\circ}$ faire succéder les 
récoltes de manière que les unes préparent la réussite des autres; $3^{\circ}$ entre deux récoltes épuisantes (telles que les céréales), placer une ou plusieurs récoltes améliorantes (telles que les récoltes sarclées, le sainfoin, le trèfle, ete.) ; $4^{\circ}$ remplaecr les plantes qui salissent le terrain par des plantes qui l'ombragent fortement (commele sarrasin, les pois), ou qui exigent des cultures répétécs (eomme les récoltes sarclées).

Voici un exemple d'assolement par rotation :

1ère annéc..........Racines sarelées et fumées, ou jachère.

$2 \mathrm{c}$ " Céréales avec graine de foin.

3e " Foin.

te $:$ Foiu.

5c " Pâturage.

6e " Avoine ou pois.

13. Pourquoi faut-il ćgoutter les terres, et comment faut-il le faire?

Il faut égoutter les terres, parce qu'un exeìs d'humidité gêne l'action des engrais, nuit à la germination des semences, favorise les mauvaises herbes, compromet les récoltes, rend les travaux difficiles et insalubres, etc.

A part les rigoles, on fait, pour égoutter un terrain, des fossés, qui reggivent les caux surabondantes et les conduisent dans quelque ruisseau. Ces fossés peuvent être remplis de pierres, entre lesquelles l'eau s'écoule, puis recouverts d'autres pierres plus graudes et d'uno couche de terre, ce qui empêche de perdre du tcrrain, et gêue moins la circulation dans les champs. Il est eneore préférable de placer des drains, ou tuyaux en terre cuite, au foud de ces fossés couverts. Cette dornière méthode se nomme drainaye.

\section{III.}

11. Quelles sont les principales semences employées?

Les principales semonces dans les champs sout : $1^{\circ}$ les céréules, ou plantes farincuses : le blé, le seigle, l'orge, l'avoine, le maïs ou blé-l'Inde et le sarrasin ; $2^{\circ}$ les légumes : les pommes de terre (pututes,), les caruttes, les navets, les betteraves (raeines); les choux; lis puis et bos fêve; $3^{\circ}$ les plantes textiles: le lin, lo 
chanvre $; 4^{\circ}$ les plantes fourragères : le mil, le trèfle, le sainfpin, etc.

Dans les jardins, la rave, l'oignon, le poireau, l'ail, l'éehalote, le melon, la citrouille, le tabac, sans compter plusicurs des scmences précédentes.

15. Pourquoi est.il nécessaire de bien choisir la semence ?

Il faut bien choisir la semence, pour que la récolte soit plus abondante, plus riche et plus nette.

16. Quels sont les procédés employés pour nettoyer le grain ?

On nettoie le grain avec le van et le crible. S'il est destiné à la semence, il pourrait être nettoyé même à la main.

17. Donnez les noms des principaux instruments d'agriculture ?

Les principaux instruments d'agriculture, ou aratoires, sont : 10 la charrue, pour labourer, c'est-i-dire pour couper une bande de terre et la renverser (quand la charrue n'a pas d'avant-train, on l'appelle araire); $2^{\circ}$ la herse, pour ameublir le sol, le mélanger avec les engrais et les amendements, arracher les mauvaises herbes, et recouvrir la semence; $3^{\circ}$ le rouleau, pour écraser les mottes de terre et donner de la consistance au terrain; $4^{\circ}$ la houe à cheval, composée de socs et de couteaux et destinée à détruire les mauvaises herbes et à ameublir la surface du sol ; $5^{\circ}$ le buttoir, composé de deux versoirs et destiné à chausser (renchausser) les plantes $; 6^{\circ}$ le semoir, pour semer par rangs ; $7^{\circ}$ la faucille et la faulx, pour couper le grain et le foin ; $8^{\circ}$ le féau et le moulin à battre, pour sépurer le grain de l'épi.

18. Quelle est l'importance d'avoir de bons instruments pour cultiver?

Il est important d'avoir de bons instruments pour cultiver, paree que c'est le moyen d'exécuter les travaux plus promptement, plus commodément et d'une manière plus parfaite, et par là même de retirer plus de profit de la eulture, pourvu que ees instruments ne soient pas trop coûteux, a raison de la culture que l'on a à faire.

19. Quels sont les principaux travaux agricoles?

Les principaux travaux agricoles sont: le défrichement, Io transport du fumier, le labour, les semailles. le hersage, le binage, 
le sarclage, le buttage, le fauchage, la coupe du grain, l'engrangement, le battage, l'entretien des clotures, des fossés et des chemins.

20. Dans quel temps les travaux agricoles doivent-ils se faire? Le défrichement du terrain boisé se fait soit le printemps, soit l'automne et l'hiver, afin de faire brûler l'abatis l'êté suivant. Le labour s'exécute le printemps aussitôt que l'état du terrain le permet : on doit surtout en faire l'automne autant qu'on peut. Les grains se sèment du milieu d'avril au commencement de juin ; aussitôt après que la semence est déposée en terre, on passe la herse, puis le rouleau. Pendant que certaines plantes poussent, on bine pour ameublir la terre; on sarcle pour détruire les mauvaises herbes; on butte pour ramener la terre au pied des plantes qui exigent ces soins. La fenaison se fait lorsque le foin est en fleur. Le grain se coupe aussitôt que le pied de la tige jaunit, depuis le milieu d'août a la fin de septembre : il se bat l'automne et l'hiver. En octobre, on récolte les patates, les bettcraves, les carottes, les navets et les oignons ; en novembre, les choux d'hiver. Les réparations à faire aux clôtures, fossés et chemins s'accourplissent dans les temps où les autres travaux pressent le moins, particulièrement entre les semailles et les foins.

21. Indiquez le meilleur moyen de faire un bon labour.

Pour qu'un labour soit bon, il faut que les sillons soient bien droits, que les bandes de terre soient convenablement retournées, d'une épaisseur uniforme et d'une bonne largeur ; enfin qu'il soit exécuté à une profondeur proportionnée à la nature des plantes qu'on veut semer et à celle dụ sol et du sous-sol.

22. Faites voir les avantages qu'il y a ì bien labourcr un champ.

Les prineipaux avantages qu'il y a à bien labourer un chanip sont : $1^{\circ}$ le renversement et l'ameublissement da sol, de maniùre à permettre à la chaleur et à l'humidité d'y pénétrer, et aux racines de s'étendre pour trouver la nourriture qui leur est nécessaire; $2^{\circ}$ l'amélioration du sol par le mélange des engrais et assez souvent par l'augmentation de la couche arable (propre a la culture) $3^{\circ}$ la destruction des mauraises herbes. 
23. Intlique\% la manière de faitre la culture des légnmen.

l'ur cultiver les légumes, il faut : $1^{\circ}$ un sol plutôt léger que fort ; $2^{\circ}$ plusieurs lal ours, dent un au moins profond : $3^{\circ}$ suffisuirment d'ongrais ; $4^{\circ}$ piendant la régétation, binage et pour phusicurs du butt:ge. 11 ast ncore préférable de scmer par rangées.

\section{IV.}

24. Indignez le ne $m$ des n:auvaises herbes les plns cemmunes et le moyen de les détruire.

Les matwaises licibes les filus comnunes sont : le chardon, la marguerite, le chiendent, le plantain, la chicoréc sauvage, l'oseille, la ctmomille, la fougire, etc.

Le nocycu de les détruire est d'arracher ces plantes avant qu'elles ne portent graine, par des labours et des sarelages répétés.

25. Faites voir la nécessité de la culture des herbes.

Les herbes sont fort utiles comme assuisonnement, plusicurs niême comme remìdes.

206. Quelles sont les herhes le plus cultivées dans le pays ?

l.es herbes le plus cultivées dans le pays sont: le persil, le cerfeuil, la sariette, la ciboule, ete.

27. Donnez le nom des principaux aninaux domestiques.

les prineipanx animaux domestiques utiles aux enltivateurs, or ininaux de ferme, sont: le cheval, le boeuf, la vache, le porc at le mouton.

2X. Quelle est la quantité relative d'animaux que le cultivateur dnit álever?

Le enltivateur doit élever un nombre d'animaux proportionné à l'itendue de ses travaux, al la nourriture qu'il peut leur donner pour les conscrver en bon ordre, et à la quantité de fumicr qu'il lui fant.

29. Quel est le but de l'umélionation des races ?

On doit travailler à l'amélionation des races dans le but d'avoir des animaux plus propres aux travaux auxquels on les destine, qui donnent de meilleure viands et en plus grande quantite, et, pour les moutons, plus do laine; pour les vaches, plus de lait. 
30. Dass quel eas le croisement des races peut-il être avantagéux ?

Le eroisement des races est avintugeux lorsque les animaux reprodueteurs conviennent bien. au pays et au elimat, et qu'ils sont d'une espèce dont l'entretien n'est pas trop coûteux.

31. Quels sont les moyens a prendre pour faire de bon beurro ?

Las moyens a prendre pour faire de bon beurre sont : $1^{\circ}$ d'être trèss-propre; $2^{\circ}$ de uo pıs laisser trop vieillir la erême ; $3^{\circ} \mathrm{d}^{\mathrm{e}} \mathrm{e}$ bien exprimer tout lo lait $: 4^{\circ}$ de le saler convenablement.

32. Quels sont les arbros fruitiers les plus cultivés diuns lo pays?

Las arbres fruitiers les plus cultivér dans lo pays sont: le pommier, le prunier, lo poirier et le corisior ; les arbustes : lo groseillier, le gadelier et le framboisier.

33. Quals sont les soins qu'ils faut donner aux arbres fruitiers en général ?

Las soius a donner aux arbes fuitiers en géníral sont : de les nattoyer (ôter les branches inortes), de les triller (enlever les branches nuisibles), de les greffer (pour avoir des produits plns ba:ux et moillours), do les échouiller, enfin do les mettre ì une distance convenable. 


,




\section{REPONSES}

$\Delta U$

\section{PRAORAIIIII}

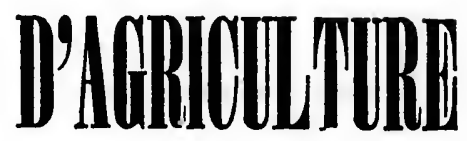

POUR LI

\section{DIPLOME D'ACADÉMIR}

\section{URDULE H. No. 8}

\section{I.}

1. Objet et arantages des ótudes agriooles.

Les études agricoles ont pour objet de faire connaitre la meilleure manière d'exécuter les différents travaux de la oampagne, d'après l'expórience des plus habiles cultivateurs, et pour avantages de faire obtenir les produits les plus beaux et les plus riohes areo le plus de profit possible.

3. Conditions nécossaires pour que la germination se fasse bien.

Pour que la germination se fasse bien, il faut : $1^{\circ}$ que le grain wit bien ohoisi et assez noureau; $2^{\circ}$ que le terrain lui convionne; $3^{\circ}$ qu'il soit enterré et espacé convenablement ; $4^{\circ}$ que les conditions d'air et d'bumidito noient farorablen. 
3. Noms des différentes espices de terre.

Il y a trois especes principales de terre: $1^{\circ}$ les terres argileuses, formées d'argile ou glaise; $2^{\circ}$ les terres silicenses, formées de silice; $3^{\circ}$ les terres calcaires, composées de curbonate de chaux. Si ces substances sont mélangées, comme elle le sont presque toujours, le nom varie, et le terrain s'appelle argilosiliceux, silico-argileux, etc., selon que l'argile, la siliee, etc., y domine.

4. Noms des substances qui composent un sol : indiquer celles qui donnent de bonnes qualités a la terre.

On appelle terre forte celle où donine la glaise ou argile; elle est tenace et froide; on la reconnaît a ce qu'elle se crevasse a la sécheresse, et que l'eau séjourne a sa surface. I Les avantages en sont : qu'elle garde mieux la frâtcheur, offre aux racines une basc plus solide, et conserve plus longtemps la richesse que lui ont communiquée les engrais. Fn revanche, elle retient trop l'humidité dans les temps de sécheresse. Le blé, l'avoine, les fives et les betteraves y viennent bien, ainsi que le trèfle.

On appelle terre légère celle qui est formée surtout de sable ou de carbonate de chaux (matière dont on peut extraire de la chaux). Les avantages d'un terrain sablonncux sont : qu'il se ressuie pl us vite, que les plantes y lèvent et y mûrissent plus tôt, et que les cultures y sont faciles et moins coûteuses. Les inconvenients en sont : de s'assécher trop rapidement, de retenir peu les matières fertilisantes, et de trop exposer les plantes aux variations bruaques de température. L'orge, le seigle, le sarrasin, les navets et la poinme de terre (patate) y réussissent particulièrement.

Les avantages du culcaire dans le sol sont : de rendre les terres fortes plus meubles, plus friables, et conséquemment plus fueiles a cultiver, et de donner aux terres légìères plus de consistance et par lì même d'en faciliter aussi la eulture. Le earbonate de chaux d'ailleurs améliore la qualité de certains produits. L'orge et le sainfoin réussissent bien dans les terrains caleaires.

Enfin, la meilleure terre ou terre franche, est celle qui renferme de l'argile, de la silice (sable et civilloux) et du carhounte: de chaux en proportions convenables. avec enviruu un clonzicuse 
d'humus ou terreau. On appelle ainsi une substauce brune ou noirâtre produite par la décomposition des matières animales ou. végétales.

5. Influence du sous-sol sur la bonté d'une terre ainsi que la pente du sol.

Le sous-sol, e'est-d-dire la eouehe qui vient immédiatement après le sol arable, étant ramené plus ou nooins à la surface et melangé avec le sol par des labours de plus en plus profonds peut influer beaucoup, d'après sa nature, sur la bonté d'une terre.

La pente du sol a également un effet avantageux ou nuisible par rapport : $1^{\circ}$ à l'écoulement des eaux; $2^{\circ}$ a l'exécution des travaux $; 3^{\circ}$ à l'éboulement des terres $; 4^{\circ}$ à l'exposition, ou a la partie de l'horison vers laquelle il ineline.

\section{II.}

6. Moyens d'amélioration d'une terre.

1. Le défrichement, qui consiste à mettre en état de culture, soit un terrain abandonné, soit un bois ;

$2^{\circ}$ l'épierrement, qui eonsiste a débarrasser le terrain des pierres dont il est encombré ;

$3^{\circ}$ l'écobuage, qui eonsiste ì enlever par tranches la croûte supérieure du sol et à la brûler ;

$4^{\circ}$ l'assainissement, qui consiste à délivrer le sol des eaux surabondantes ou stagnantes;

$5^{\circ}$ enfin, les amendiments, qui eonsistent dans le mélange avce le sol de eertaines substanees qui (eomme la chaux, la marne silicense et le sable) le rendent menble, s'il est trop enmpacte; ou qui (comme l'argile, lit marne argileuse et la.chaux) le rendent. plus ferme, s'il est trop meuble.

7. Ce qu'on entend par assolement ; prineipe des assolements.

On appelle assolement l'ordre dans lequel se suecèdent les diverses productions d'un même terrain : il peut être de trois, de quatre... de huit, de nenf ans, ete. Cctte succession cle produits est bien néeessuire, parce que, parmi les plantes, les unes serrent à ameublir le sol, d'autres à le nettoyer; quelques-unes sont améliorantes, d'autres épuisantes, ete. Il faut que l'assolement ait pour effet de rendre au sol ce qu'on li:i a enleré. 
Les assolements sont appuyés sur le principe que chaque espèce de plantes absorbe des sues particuliers, de manière que le sol s'appaurrit graduelkmont, lorsqu'cn y cultive longtcmps les mêmes plantes.

8. Ce qu'on entend par cngrais organiques; les principaux.

On entend par engrais organiques ceux qui proviennent de la décomposition des matières soit animales, soit végétales. Les principaux sont : les exercments, les urines, les os, le fumier et les cadarres des animaux, les débris végétaux, les varees, les cendres, etc.

Voici les différentes matières qui peurent surtout servir d'engrais (substances destinées à enrichir le sol) : $1^{\circ}$ certaines plantes qu'on enfouit avant qu'elles ne soient mûres, ce qu'on nomme engrais verts, ou engrais végetaux; $2^{\circ}$ les tourteaux qui viennent du lin, après qu'on en a extrait l'huile; $3^{\circ}$ les excréments et les urines, qu'on appelle engrais animaux : $4^{\circ}$ les fumiers d'etable, ou engrais mixtes; $5^{\circ}$ les cendres; $6^{\circ}$ les composts, formés de chaux et de marne mêlées par couches avec des débris de toute nature.

9. Donner des détails sur la bonté relative des engrais ainsi que sur la manière de les appliquer.

Il faut distenguer les fumiers chauds d'avec les fumiers froids, parce que les uns et les autres ne conviennent pas également d toutes les espèces de terrains. Les preniers (excréments de l'homme, des volailles, des chevaux et des moutons) conviennent aux terres fortes et froides; les seconds (excréments des bêtes a cornes), aux terres sablonneuses et légères.

Les engrais liquides, tels que l'urine et le purin (eaux de fumier) paruissent être les engrais les plus actifs, mais leur effet n'est pas de longue durée. Viennent ensuite les engrais minéraux solides ; puis le fumier d'étable, enfin les engrais verts.

Les fumiers enfouis frais agissent pius lentement, mais leur action est plus durable.

Quant a l'application do l'engrais, il n'est pas ordinairement d propos de le laisser sur le champ par petits tas; il vaut mieux l'étendre et l'enterser imnédiatement. On doit en mettre plus 
sur les hauteurs yue sur les parties basses du terrain. La quantité à répandre dépend de la nature du sol, de celle des plantes à cultiver et de la qualité de l'engrais.

10. Dans quelles limites le fumier doit-il aroir fermenté pour qu'il soit le meilleur possible?

Le funier qui éproure une fermentation très-active, perd une grande partie de ses principes fertilisants. Il est done désirablo que le fumier soit sotmis it une fermentation lente et égale dans toutes les parties du tas. On doit pour eela l'arroser fréquemment.

11. Ce qu'on entend par engrais minéraux; terrains où la chaux peut être alplicquée avec avantage ; emploi du plâtre.

On entend par engrais minéraux ceux qui ne proviennent ni des animaux, ni des végétaux : tels sont le plître, la chaux, la marne, ete. La chaux, qui s'emploie surtout pour amender lo sol, peut être appliquée avec avantage, soit aux terrains argileur pour les ameublir, soit aux terrains siliceux pour leur donner plus de consistance. Le chaulage se fait it dose plus ou moins forte, suivant le besoin. Le plître cuit ou cru s'emploie ou comme engrais, en l'appliquant directement au sol dans les prairies artificielles, ou coınme stimulant, en le répandant a la rolée sur les plantes en végétation. Dans tous les cas, il faut qu'il soit bien pulvérisé.

12. Le but qu'n doit se proposer dans l'amélioration des races; moyens qu'on peut employer.

On doit travailler il l'anélioration des raecs dans le but d'avoir des animaux plus propres anx travaus auxquels on les destine, qui donnent de meilleure riande et en plus grande quantité, et pour les moutons, jlus de laine ; pour les vaches, plus de lait.

Pour parvenir it anéliorer les rnces, il serait important d'avoir dans chaque enuté, de beaux animaux reproducteurs, de taille moyenne, venant de pays ì peu près semblubles au nôtre, et que les cultivateurs pussent avoir a leur disposition.

13. Soins qu' oul doit mettre dans le choix des parents pour lo croisement des races. Soins hygieniques qu'on doit donner aúx nuimaux. 
Le croisement des races ent avantageux lorsque les animaux reproductcurs conviennent bien au pays et au climat, et qu'ils sont d'une espèce dont l'entretien n'est pas trop coûtenx.

Les parents doivent être de plus des animaux sains, robustes et bien constitués.

Les animaux domestiques doivent être traités avec douceur, recevoir une nourriture saine, abondante et bien réglee, être tenus propres ; enfin, n'être point soumis à un travail exeessif ou inutile. L'habitation où on les loge, doit être suffisamment spacieuse, élerée, sèche et aérée. Les urines doivent pouvoir $s^{\prime}$ ccouler facilement dans un réservoir, ou'dans le trou au fumier.

Le nombre d'animaux qu'il faut élever dans une ferme, dépend de la quantité de nourriture qu'on peut leur donneer, et de celle du fumier dont on a besoin. En général, il vaut mieux en a voir moins, et les nourrir convenablement. La paille qu'on leur donne devrait être haohée avec l'instrument appele hache-paille.

Les chevaux doivent être tenus tress-propres, et ne travailler que selon leurs forees.

Il est a propos d'enlever les fumiers des bêtes a cornes tous les trois ou quatre jours, et de répandre chaque jour, sur la litiere de la veille, une litière nouvelle.

Les moutons doivent être renfermés dans des bergeries bien aérées, construites sur un terrain see, non pavées et sans égout. Il est bon de saler leur nourriture.

Les cochans doivent pouvoir prendre l'air et s'ébattre dans de l'eau, auprès de leur loge. Lorsqu'ils prennent leur nourriture plusicurs ensemble, il faut disposer leur auge de manière qu'ils ne puissent se quereller.

\section{III.}

14. Soins qu'on doit mettre dans le choir d'une terre, et grandeur relative de cette terre.

Quand il s'agit d'acheter uns terre, on doit en choisir une d'une grandeur proportionnéc aux moyens dont on peut disposer, au nombre de bras qu'on peut employer, et a l'espece de culture que l'on a en vie. On doit preférer unc terre dont l'exposition snit au sul, cui puisse s'assainir facilement, dont lo sol ne soit ni 
paurre, ni épuisé, ni trop humide, ni trop léger, qui ne présete point de pentes trop raides, qui fournisse assez d'eau, qui ne soit pas trop coupée ou mangée par des ruisseaux, enfin, sur laquelle il reste ssez de bois. La distance du marehé, l'éloignement de l'église et des moulins, la facilité des communications, l'état des chemins, doivent aussi être pris en considération.

15. Nom̉s des bâtises nécessaires à un fermier, de leur disposition.

10 La demeure du fermier, qui doit être construite dans un endroit sain, sufflsamment éloignée du chemin, protégée contre le vent, et convenablemant ombragée ;

$2 \circ$ la grange, pour resevoir lo grain et le fourrage : elle comprend : $1^{\circ}$ le grenier proprement dit, pour le grain; $2^{\circ}$ l'aire, ou on le bat; $3 \circ$ le fenil, pour le foin.

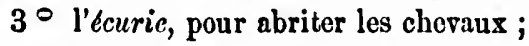

$4^{\circ}$ le hangar, ou l'on met a couvert les voitures, les instruments aratoires, et le bois de chauffage ;

$5^{\circ}$ l'étable, destinée à recevoir les bêtes à cornes ;

$6 \circ$ la bergerie, où se réfugient les moutons ;

$7^{\circ}$ la porcherie, où l'on gardo les pores ;

$8^{\circ}$ le poulailler, pour les volailles de basse-cour ;

$9^{\circ}$ le fournil, qui renferme le four ;

$10^{\circ}$ la laiterie, ou l'on conserve le lait.

Ces diverses bâtisses doivent être disposées de manichro que lo fermier puisse les surveiller toutes a la fois, qu'olles soient a la proximité de la maison, ainsi que do l'eau potable; et que la mauvaise odeur ne puisse nuire ni aux animaux, ni au lait, ni au grain, ni au foin.

Si la ferme est bien étenduc, il scra quẹlquefois nécéssaire de multiplier les granges, ou do les romplacer par des meules, ou encore micux des gerbiers.

13. Noms des principaux instruments d'agriculture avec la description des principales parties.

Les principaux instruments d'agriculture, ou aratoires, sont : $1^{\circ}$ la charrue, pour labourer, c'est-i-dire pour coupor une bando de terre et la renverser (qumd la charrue n'a pess d'avant-train, 
on l'applelle arcire); $2=$ la herse, pour ameubli. le sol, le mélanger avec les engrais et les aroendements, arracher les mauvaises herbes, et recouvir la senence; $3^{\circ}$ le roulcau, pour écraser les mottes de terre et donner de la consistance au terrain; $4^{\circ}$ la hone a cícral, composíe de eocs et de couteaux, et destinée a détruire les nauvaises herbes et a ameublir la surface du ol ; $5^{\circ}$ le buttoir, composé de deux versoirs et destiné a chausser (renchausser) les plantes $; 6^{\circ}$ le semoir, pour semer par rangs; $7^{\circ}$ la faucille et la faulx pour couper le grain et le foin; $8^{\circ}$ le fléau et le moulin à buttre your séparer le grain de l'épi.

Dans la charrue on distingue le joug, pièce de bois placée sur la tête des bœufs pour les atteler ; l'avant-train, composé d'un essicu et de roues (l'araire n'en a point) ; le coutre, espece de fort couteau, en avant du soc, destinć a trancher la terre verticalement; le soc, qui coupe et soulève la terre horizontalement, et comprend laile, qui est une lame d'acier, et la douille, qui l'assujettit au corps de la charrue; le sep, sur lequel elle repose, et qui glisse sur le fond du sillon; le versoir ou l'oreille, qui retourne la terre ct la renverse sur la raie roisine; l'âge, flèche ou haie, pièce de bois a laquelle tiennent les autres parties de la charrue; les étangons, qui relient le sep à l'âge : le régulateur, qui sert à régler lit largeur et l'épaisseur de la tranche de terre; enfin, les mancheroms, placés à l'arrière do l'instrument, et par lesquels le liboureur le dirige.

Ta herse se compose d'un e'. îssis de bois, garni de dents ; cellesci doivent être disposées de manière à bien diviser toute la surface du terrain.

17. Nécessité de l'úgouttage d'une terre ; moyens à employer pour y arriver.

Il faut éggoutter les terres, parce qu'un excès d'humidité gêne. l'action des engrais, nuit à la germination des semenees, favorise les mauvaises herbes, compromet les récoltes, rend les travaux difficiles et insalubres, etc.

A part les rigoles, on fitit. pour egoutter un terrain, des fossés, qui reçoivent les eaux surabondantes et les conduisent dans quelque ruisseau. Ces fossés penvent être remplis de pierres, entre 
lesquelles l'enu s'écoule, puis recouverts d'autres pierres plus grandes et d'une couche de terre, ce qui empêche de perdre du terrain, et gêne moins la circulation dans les champs. Il est encore préférable de placer des drains, ou tuyaux en terre cuite, au fond de ces fossés couverts. Cette dernière méthode se nomme drainage.

18. Les conditions d'un bon labourage, grandeur de la tranche et sa hauteur, forme et grandeur des planches.

Pour qu'un labour soit bon, il faut que les sillons soient bien droits, que les bandes de terre soient convenablement retournces, d'une épaisseur uniforme et d'une bonne largeur ; enfin, qu'il soit exécuté $\mathfrak{i}$ une profondeur proportionnée à la nature des plantes $q u$ 'on veut semer et a celle du sol et du sous-sol.

Si toute la surface d'un champ a été labourée de manière à ce qu'elle soit parfaitement unie et non coupée de sillons ou raies d'écoulement, on ditque le labour est ì plat. Il est par planches, lorsqu'on a ménagé, de distance en distance, des raies d'égouttement paralleles. Enfin lorsque les sillons d'écoulement sont plus rapprochés les uns des autres, ot que la planche est plus ou moins bombée, on dit que le champ est labouré en billons.

19. Epoque où doivent se faire les labours ; raisons du choir, but du hersage.

Le moment le plus favorable aux labours est celui où la terre n'est ni entièrement sèche, ni tout-à-fait humide : alors en effet elle se brise et s'émiette naturellement en se retournant.

Le hersage qui suit le labour, a pour but de pulvériser les mottes soulevées par la charrue, de recourrir la semence, et de mêler plus complétement les différentes parties du sol.

20. Ce qu'on entend par rotation, systèmes de rotation les plus employés.

On entend par rotation, la succession des récoltes selon un ordro détcrminé dans les différentes parties d'une terre, de manière que la culture parcoure comme un cercle régulier, qui ramène les mêmes récoltẹs au bout du même nombre d'années. 
Voici un exemple de rotation:

1ère année ...........Plantes sarclées et fumées, ou jaolìre;

\begin{tabular}{|c|c|c|}
\hline $2 \mathrm{e}$ & " & .......... Céréales avec graine de foin \\
\hline $3 e$ & “ & …........Foin. \\
\hline 4 & " & ........... Foin. \\
\hline 5 e & " & .......... Foin. \\
\hline $6 \mathrm{e}$ & " & ...........Pâturage. \\
\hline & " & ...........Pâturage. \\
\hline & " & ............Pois. \\
\hline 9 & “ & ..........Avoine. \\
\hline
\end{tabular}

21. Ce qu'on entend par jachères; leurs avantages, manidre de les pratiquer.

On entend par jachère un temps de repos, ou de non produetion, accordó au sol. Elle est nécessaire lorsque le cultivateur ne peut se proourer l'engrais que le sol réclame, ou eneore lorsqu'il faut détruire les mauvaises herbes par des labours donnós pendant l'ét6. Mais un système intelligent d'assolement remplace la jachdre avec avantage.

\section{IV.}

22. Soins d donner au choix des stmences; avantages qn'il J a des changer.

Les graines que l'on veut semer, doivent etre bien mûres et récoltées sur les plants les plus vigoureux. Si elles sont luisantes et renflées, o'est un indice qu'elles sont saines et bien formóes.

Elles ne eonservent ordinairement leur faculté de germer qu'un nombre plus ou moins grand d'ennées.

L'expérience paraît prouver qu'il est avantageux de changor les semences, et que la plante qui se produit toujours dans lo même sol, vient à dégénérer.

233. Noms des plantes qui sont les plus cultivées en Canada ; avantage de faire la moisson avant la maturité.

Les prineipales semences dans les champs sont : $1^{\circ}$ les céréales, ou plantes farineuses : le blé, le seigle, l'orge, l'avoine, lo maïs ou bléd'Inde ct le sarrasin ; $2^{\circ}$ les légumes : les pommes de terre (patates), les carottes, les navets, les betteraves (racines); les choux, los pois et leis fives: $3^{\circ}$ les plantes textiles: lc lin, le 
chanvre $4^{\circ}$ lew plsntes fourrugires : le mil, le trèfle, le sainfoin, etc.

Dans les jardins : la rave, l'oignon, le poireau, l'ail, l'echalote, le molon, la citrouille, le tabao, sans compter plusieurs des semenecs préeédentes.

Il est utile de réeolter le grain avant qu'il soit complétement mâr, parce qu'on évite par la l'egrenage du grain et son exposition trop longue a l'intempérie des saisons, et que, s'il paraît moins pesant pour le moment, il reprend bientôt de l'avantage quand il w'est durci lentement dans la grange.

24. Terrains qui conviennent le mieux a la culture du bl6; époque de la semaille.

Le ble demande un sol plus argileux que sableux, ayant une certaine consistance, et abondant en humus. Le blé d'automne me sème dans le mois d'aout, et celui du printemps au commencode mai.

Il est avantageux de chauler le blé que l'on veut semer, c'estd-dire de le passer à l'eau de chaux.

25. Culture du seigle, oulture de l'orge: terre qui convient le mieus d la cultare de ce grain ; pratique de la culture de l'orge.

Le seigle sè contente d'une terre leggère et peu riche; il se récolte deux ou trois semaines avant le blé. Sa paille sert da couvrir les bâtiments et a lier les gerbes, mais donne un mauvais fourrage. Il y a deux especes de seigle : le seigle de printemps et celui d'automne.

L'orge demande une terre fraîche, meuble et plus riche que pour le seigle. Les terrains caleaires lui conviennent bien. Ello doit être enterrée plus profondément que le blé, et serrée bien sèche.

26. Culture de l'avoine, ses avantages.

La culture de l'avoine demande moins de soins que celle des autres céréales, et cette plante est peu difficile sur le choir du terrain. Cependant ses produits sont doublés par nne culture bien entenduc. En général, un seul labour lui suffit. Il est important de la semer do bonne heurc. 
27. Pratique de la culture du maïs ; ses usages.

Le maïs ou blé-d' Inde préfère une terre légère et humide. Si l'on veut récolter la graine, on le sème en lignes; si on le cultive pour fourrage, on le sème à la volée. Dans le premier cas, on lui donue deux ou trois binages et buttages. Il faut aussi ôter avec soin toutes les pousses latérales, afin que l'épi soit plus gros. On coupe encore le haut de la tige. après la floraison, pour la donner en vert aux bestiaux. Le fourrage qui provient du mais est excellent.

Quand on veut récolter la graine, on cucille le maïs en cassant le pédoncule (pied) des épis, lorsque ceux-ci sont mûrs, ce que l'on reconneît à la couleur et a la dureté du grain. On les fait sécher; puis on arrache les feuilles, et on laisse eneore les épis séeher. Dans le cours de l'hiver, on égrène le maìs à la main ou avec un instrument.

28. Sols qui conviennent le mieux aux pois; manière de les cultiver.

Toute espèce de terre convient à la culture des pois ; le fumier leur est nuisible, la chaux au contraire leur est très-utile. Ils demandent des labours profonds. Pour les récolter dans les champs, on n'attend pas que toutes les graines soient mûres. On peut aussi les donner aux animaux comme fourrage vert, en les fauchant avant leur maturité.

29. Pratique de la culture de la pomme de terre; terres qui lui sont les plus propices.

La pomme de terre (patate) préfèrc en général les terres légères; elle exige des labours profonds et des fumicrs abondants. On peut en semer la graine ou planter les tubereules; dans ce dernicr eas, il paraît être plus avantageux de choisir les plus petits, pourvu qu'ils soient bien mûrs, et de les planter enticrs. On les plante ì la bèche ou avec la charruc. La pomme de terre demande des hersages ct des buttages. Elle doit se récolter lorsqu'elle est parvenue à sa maturité complète, qui s'annonee par lo dessèchement des fanes. L'arrachement se fait à la main ou avec la charrue. Pour conserver les pommes de terre pendant l'hiver, 
il faut les préserver de la gelée, de l'humidité, qui les ferait pourrir, de la chaleur et de la lumière, qui les feraient germer.

Elles sont très-utiles pour la nourriture dès bestiaux : crues, elles augmentent la quantité du lait; cuites, elle engraiesent beaucoup les animaux; un pou formentées, elles les engraissent encore plus.

30. Manière de cultiver les carottes et les navets; leurs usages.

La carotte se plaît dans les terres meubles, fraîches et légères ; comme elle est pivotante, elle exige des labours très-profonds. Le navet prefère des terres compactes, profondes et bien préparés. Ces deux plantes demandent $a \hat{~ e ̂ t r e ~ s u f f i s a m m e n t ~ e s p a c e ́ e s, ~ s e m e ́ e s ~}$ dans un terrain bien fumé, herées, sarclées et binées. La graine de navet, étant très-petite, doit être peu enterrée.

Ces produits crus, ou mieux cuits, forment une excellente nourriture pour les animaux. On les tranche avec un instrument appelé coupe-racines.

31. Manière de semer le trèfle, de le récolter; ses avantages.

Le trèfle se sème seul, ou mieux avec une céréale de printemps; la graine doit être très-légèrement enterrée. La terre doit être préparée par de bonş et profonds labours. Il est avantageux de répandre sur le trèfle qui pousse, du plâtre comme stimulant. On doit récolter le trèfle l'année après l'ensemencement ; on le fauche lorsque la floraison est complete. On ne doit pas le faner comme le foin, mais seulement retourner les andains. Les trèfles réservés pour graines se coupent quand ils sont parfaitement mûrs.

Le trèfle fournit un fourrage abondant et fort recherché des bestiaux; il améliore le sol par le repos qu'il lui procure, l'ombrage qu'il lui donne, et les détritus de ses feuilles et de ses racines ; enfin, dans la rotation des récoltes, il se place avantageusement sur du blé et avant de l'avoine. Il est encore une bonne préparation pour une récolte de pommes de terre ou de racines sarclées.

32. Détails sur la culture du foin, emploi le plus convenable qu'on doit on faire.

Lo foin doit we faucher lorsqu'il est en fleur; il s'étend sur la 
terre en andains. Le lendemain, lorsque la rosée est disparue, on le fane en retournant les andains avec une fourche de bois, ce que l'on continue jusqu'd ce qu'il soit bien sec. Le soir, on le ramasse par petits tas avec le râteau. Quand le foin est assez sec, on le met en veillottes et on le transporte au fenil, ou bien on le conserve en meules bien faites. On peut aussi le botteler. L'air doit pouvoir circuler autour du foin placé dans la grange:

35. Animaux qu'il faut elever dans une ferme ; soins à donncr de chacun d'eux.

Il est important, pour un caltivateur, d'elever un nombre suffisant d'animaux pour leur faire exécuter les travaux de la ferme ou pour les vendre. Dans le premier cas, il faut choisir les plus beaux produits, qui soient sains et bien conformés; dans le second, avoir grand soin de les engraisser d'une manière al la fois prompte et économique.

Le jeune cheval ou poulain ne doit guère travailler avant l'âgo de deux ans et demi ou trois ans, et être ménagé au moins jusqu'd quatre ans. Il ne doit être accoutumé au travail que peu a peu et avec douceur, et n'être ferré que le plus tard possible.

On fait d'abord têter les veaux, ou on leur donne autant de lait qu'ils en peurent prendre; plus tard, on y ajoute des oufs, et une sorte de bouillie faito aveo de la farine et du lait ; puis des raves, des pommes de terre ou des carottes érasées en purée, aveo de l'cau et du lait.

34. Description d'une bonne laiterie; maniere de faire le beurre.

Une bonno laiterie doit conservor en tout temps une tempéra. ture égale, c'est-d-dire, une oertaine fraîcheur en été, et une oertaino chaleur en hiver. Il est micux qu'clle soit voatée, et que le sol soit pavé en pierres. Los vases so mettent sur des tablettes, toujours tenues bien propres. Dans une sorte de restibule on les lave et on les échaude.

Pour faire le beurre, on met le lait, après l'avoir coulé, dans des vases qui prósentent boaucoup de surface et peu do profon'deur. On l'écrême, et on conserve ensuite la crême ainsi obtenue 'dans un vase d part, jusqu'd ce qu'on en ait assez pour faire le 
beurre. On se sert d'une baratte pour battre la crême, et on reconnaît que le beurre est fait quand il tombe par grains ou par petites masses au fond de la baratte. On opère alors le delaitage, c'est-d-dire, qu'on jette le beurre dans des vases remplis d'eau frâ̂che, et qu'on le presse à l'aide de cuillères plates de bois ou de battoirs, afin de le séparer complétement du lait de beurre. On le sale arec soin, et oa le recourre de saumure.

35. De la fibrication du fromage.

Pour faire du fromage, on fuit d'abord cailler le lait, ordinairement au moyen de présure; puis on sépare le caillé d'avec le sérum jusqu'd ce que lo promier prenne de la consistance; on le sale, on le met dans des formes ou on le presse, et on le renverse ensuite sur des claies courertes de paille, ou on a moin de le retourner souvent.

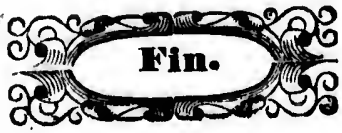

